\title{
Searching for Better Randomized Response Schemes for Privacy-Preserving Data Mining
}

\author{
Zhengli Huang, Wenliang Du, and Zhouxuan Teng \\ Department of Electrical Engineering and Computer Science, \\ Syracuse University, Syracuse, NY 13244, U.S.A. \\ \{zhuang, wedu\}@ecs.syr.edu, zteng@syr.edu
}

\begin{abstract}
To preserve user privacy in Privacy-Preserving Data Mining (PPDM), the randomized response (RR) technique is widely used for categorical data. Although various RR schemes have been proposed, there is no study to systematically compare them in order to find optimal RR schemes. In the paper, we choose the R-U (Risk-Utility) confidentiality map to compare different randomization schemes. Using the R-U map as our metric, we present an optimal RR scheme for binary data, which helps us find an optimal class of RR matrices. From this optimal scheme, we have discovered several heuristic rules among the elements in the optimal class. We generalize these rules to find optimal class of RR matrices for categorical data. Based on these rules, we propose an RR scheme to find a class of RR matrices for categorical data. Our experimental results have shown that our scheme has much better performance than the existing RR schemes.
\end{abstract}

\section{Introduction}

Data mining is a technology to extract useful patterns and trends from a large sum of data. If the original information is obtained directly, many techniques, e.g. statistics, artificial intelligence, can be utilized to analyze the information. Generally, the data are collected from different individuals or data owners. The data owners, however, might not be willing to share their data to others because of privacy concerns. This motivates a field called Privacy-Preserving Data Mining (PPDM). In PPDM, the original data are disguised; with the disguised data, the data patterns included in the original data can still be extracted. This is becoming more and more important in current society, especially when privacy is becoming a prominent concern.

When data are categorical, randomized response $(\mathrm{RR})$ technique can be used to achieve the privacy-preserving data mining. The $\mathrm{RR}$ technique for binary data was first introduced by Warner [13, which can be easily extended to general categorical data 4]. In the generalized RR scheme, there is a transformation matrix $M$ (called RR matrix) of $n \times n$, for $n$ of categories of the data. With a RR matrix $M$, the original data are randomized and produce disguised data. Different $M$ 's will achieve different accuracy level (regarding data mining results) and different privacy level (regarding how much of the original data is disclosed). How to 
choose the elements of $M$ has been studied in [5]. But when the paper compares different schemes, it only compares accuracy, which is just one important aspect of a RR scheme. Because the two aspects - accuracy and privacy - are all important in an RR scheme, using any single one of them to compare different schemes is incomplete. The first goal of this paper is to find a way to compare different $\mathrm{RR}$ matrices. Once we can fairly compare different matrices, our next question is whether there is a way to find an optimal class of RR matrices; namely, given an accuracy level, we would like to generate a matrix with optimal privacy.

To compare different randomized response schemes, we use a Risk-Utility confidentiality (R-U) map, proposed by Duncan et. al [7]. R-U map is a curve describing the relationship of accuracy versus privacy when the parameters of a RR scheme change. Armed with the R-U map which can be used to compare different RR schemes, we would like to find an optimal class of RR matrices, forming a R-U map that is consistently better than the R-U maps of other RR schemes.

To find the optimal class of RR matrices, we present a deterministic algorithm to find an optimal class of RR matrices for binary data (two categories) which have the highest utility given any risk value. According to the deterministic approach for binary data, we have identified several heuristic rules from the optimal matrices. By using the rules and a constraints possibly added by the data owners, we propose an algorithm that can find a better class of RR matrices for categorical data. Our experiments show that the R-U map of the class of RR matrices generated by our algorithm is much better than the existing schemes.

The rest of this paper is organized as follows. Section 2 reviews the related work. The problem formulation is presented in Section 3 . A deterministic approach to finding the optimal RR matrices for binary data is presented in Section 4. Our algorithm to find a class of better RR matrices for categorical data is described in Section 5. Section 6 experimentally evaluates our scheme. Section 7 concludes the paper.

\section{Related Work}

Randomization is one of the major approaches in privacy-preserving data mining. There are two different randomization methods: the Random Perturbation scheme and the Randomized Response scheme.

Agrawal and Srikant propose a scheme for privacy-preserving data mining using randomized perturbation 3. This work has been extended by Agrawal and Aggarwal 2, who propose an Expectation Maximization (EM) algorithm for distribution reconstruction. Kargupta et al. point out an important issue, that is, arbitrary randomization is not safe [10. We study why and how correlations affect privacy [9].

The randomized response is mainly used to deal with categorical data and originally proposed by Warner [13]. Other works based on the randomized response can be found in 6 8 5 1112]. The work closest to our study is that of Agrawal and Haritsa [5]. 


\section{Problem Statement}

\subsection{General Randomized Response Technique for Categorical Data}

The randomized response technique is mainly applied to each individual attribute of a data set (although it can be applied to multiple attributes); therefore, in this paper, we only study one attribute. Suppose that an attribute of the original data has $n$ different categories, e.g. $\left\{c_{1}, c_{2}, \ldots, c_{n}\right\}$. The randomized response technique replaces each $c_{i}$ with $c_{j}$ with certain probability. We use $\theta_{j, i}$ to denote the probability that category $c_{i}$ is randomized to $c_{j}$, where $i, j=1, \ldots, n$. Based on the randomized response technique, we have the following equation:

$\boldsymbol{P}^{*}=M \boldsymbol{P}, \quad$ where $\boldsymbol{P}^{*}=\left(\begin{array}{c}P^{*}\left(c_{1}\right) \\ \vdots \\ P^{*}\left(c_{n}\right)\end{array}\right), M=\left(\begin{array}{ccc}\theta_{1,1} & \ldots & \theta_{1,2} \\ \vdots & \ddots & \vdots \\ \theta_{n, 1} & \ldots & \theta_{n, n}\end{array}\right), \boldsymbol{P}=\left(\begin{array}{c}P\left(c_{1}\right) \\ \vdots \\ P\left(c_{n}\right)\end{array}\right)$.

Based on maximum likelihood estimation, we can derive the following theorem (the proofs for all the theorems are omitted due to page limitation):

Theorem 1. The MLE (Maximum Likelihood Estimate) of the probabilities $\boldsymbol{P}$ or $\left(\widehat{P\left(c_{1}\right)}, \ldots, \widehat{P\left(c_{n}\right)}\right)^{T}$ is $\widehat{\boldsymbol{P}}=M^{-1} \widehat{\boldsymbol{P}^{*}}$, where $\widehat{\boldsymbol{P}^{*}}=\left(\frac{N_{1}}{N}, \frac{N_{2}}{N}, \ldots, \frac{N_{n}}{N}\right)^{T}, N_{i}(i=$ $1, \ldots, n)$ is the number of $c_{i}$ in the randomized data and $N$ is the total number of the records in the data set. It is an unbiased estimate.

\subsection{Problem Formulation}

When applying an RR scheme to the data set, both privacy and accuracy are desirable, but the two factors cannot be optimized at the same time; there are tradeoffs. If we want high accuracy, privacy must be disclosed to some extent. When different schemes have different tradeoffs, which tradeoff is better? i.e., which scheme is better? This is an optimization problem: Given a data set, find the optimal randomization scheme which can achieve the optimal performance in terms of privacy and accuracy.

There are two main challenges in solving this optimization problem. First, when comparing two different RR schemes, what does the "better performance" means? Second, in a straightforward brute-force approach, the search space for finding an optimal solution is large.

\subsection{R-U Confidentiality Map}

We choose to use Risk-Utility confidentiality map (R-U map) 7] to compare RR schemes. An R-U map is a curve describing the impact on disclosure risk and data utility when the parameters of a scheme changes. It uses risk as the $\mathrm{x}$-axis and utility as the y-axis.

By drawing the R-U maps, the comparisons between two schemes become easy. We can easily see when one scheme has better performance than the other, 
for example, when the utility is higher for one scheme than the other under certain risk range. Based on the maps, users can choose different parameter for different schemes under their risk and utility requirements. Therefore, we mainly use the R-U maps to compare different randomization schemes, and based on the maps to examine our scheme.

\subsection{Utility}

Since the probability distribution of the data set is used to conduct most data mining computations, it is reasonable to study the distribution when analyzing the utility of the data set. We use the mean squared errors to measure the difference between the estimated distribution and the original distribution.

Mean Squared Error. Let the probability of each data value is $P\left(c_{k}\right), k=$ $1,2, \ldots, n$ and the corresponding estimated probability is $\widehat{P\left(c_{k}\right)}$. The mean squared error of each probability estimator is defined as follows.

Definition 1. The Mean Squared Error (MSE) of an estimator $\widehat{P\left(c_{k}\right)}$ of the probability $P\left(c_{k}\right)$ is the function of $P\left(c_{k}\right)$, which is $E_{P\left(c_{k}\right)}\left(\widehat{P\left(c_{k}\right)}-P\left(c_{k}\right)\right)^{2}$, where $E_{P\left(c_{k}\right)}($.$) is the expected value.$

The MSE can be calculated from the following theorem.

Theorem 2. The Mean Squared Error (MSE) of an estimator $\widehat{P\left(c_{k}\right)}$ from the probability $P\left(c_{k}\right)$ is

$$
\sum_{i=1}^{n} \beta_{k, i}^{2} \operatorname{Var}\left(\frac{N_{i}}{N}\right)+2 \cdot \sum_{i=1, j=1, i \neq j}^{n} \beta_{k, i} \beta_{k, j} \operatorname{Cov}\left(\frac{N_{i}}{N}, \frac{N_{j}}{N}\right)
$$

where $\beta_{g, h}$ is the gth row and hth column element of the inverse of $M, \operatorname{Var}\left(\frac{N_{i}}{N}\right)$ is the variance of $\frac{N_{i}}{N}, \operatorname{Cov}\left(\frac{N_{i}}{N}, \frac{N_{i}}{N}\right)$ is the covariance of $\frac{N_{i}}{N}$ and $\frac{N_{j}}{N}$ and they are

$$
\operatorname{Var}\left(\frac{N_{i}}{N}\right)=\frac{1}{N} \cdot P^{*}\left(c_{i}\right)\left(1-P^{*}\left(c_{i}\right)\right), \operatorname{Cov}\left(\frac{N_{i}}{N}, \frac{N_{j}}{N}\right)=-\frac{1}{N} \cdot P^{*}\left(c_{i}\right) \cdot P^{*}\left(c_{j}\right) .
$$

We use the average MSE of $\widehat{P\left(c_{k}\right)}$ as the utility of the estimator of the probability distribution.

Definition 2. The Average Mean Squared Error (AMSE) of an estimator $\widehat{\boldsymbol{P}}$ of the probability distribution $\boldsymbol{P}$ is $\left.\frac{1}{n} \sum_{k=1}^{n} E_{P\left(c_{k}\right)}\left(\widehat{P\left(c_{k}\right.}\right)-P\left(c_{k}\right)\right)^{2}$.

When AMSE is large, the difference between the two probability distributions are large. In the Randomized Response scenario, large AMSE means that the estimation error is high so that the accuracy of the estimated probability distribution is low. We will use it as the utility criteria to study different RR schemes. 


\subsection{Risk}

There are many different risk measurements. Based on different risk measurements, the R-U maps will be different and the optimal RR scheme will be different. To measure the risk, we propose a new approach of privacy quantification, which is considered as the following estimation problem:

Problem 1. Given a disguised data set $Y_{s}=\left\{y_{1}, \ldots, y_{N}\right\}$ and certain prior knowledge of the original data $X$, adversaries would like to estimate the original value $x_{i}$ from $y_{i}$, for $i=1, \ldots, N$. How accurate can their estimates be?

The less accurate their estimates are, the higher privacy is. Therefore, to quantify privacy, we just need to quantify the accuracy of the estimates. We define an accuracy function $G$ to represent the accuracy score of an estimate (denoted by $\widehat{x_{i}}$ ) against the actual value $x_{i}$. With this function, the average accuracy score of all the estimates can be computed in the following:

$$
A=\frac{1}{N} \sum_{i=1}^{N} G\left(\widehat{x_{i}}, x_{i}\right) .
$$

We will derive the optimal value for $A$, which represents the best estimates that can be achieved by adversaries. We will then use this optimal result to quantify privacy.

We use $\widehat{X_{Y}}$ to represent this estimate. However, according to the randomized response technique, the same value $Y$ might be the disguised results of different values from $C=\left\{c_{1}, \ldots, c_{n}\right\}$. To maximize accuracy, given a specific $Y$, adversaries would like to find an estimate $\widehat{X_{Y}}$, such that the expected accuracy score is maximized. The expected accuracy score can be computed using the following formula:

$$
E_{X}\left[G\left(\widehat{X_{Y}}, X\right) \mid Y\right]=\sum_{X \in C} G\left(\widehat{X_{Y}}, X\right) \cdot P(X \mid Y) .
$$

This is actually the Bayes Estimate, the theory of which not only provides optimal estimates for a variety of accuracy functions $G$, but also provides a methodology to derive optimal estimates for an arbitrary $G$. In this paper, we study one specific accuracy function:

$$
G(\widehat{X}, X)= \begin{cases}1, & \text { when } \widehat{X}=X \\ 0, & \text { otherwise }\end{cases}
$$

The intuitive meaning of the above function says that when an estimate is correct, the score is 1 ; otherwise, the score is 0 .

Theorem 3. For the accuracy function $G$ defined in Equation (4), the optimal estimate $\widehat{X_{Y}}$ for a given $Y$ is the MAP (maximum a posteriori) estimate, i.e.,

$$
\widehat{X_{Y}}=\operatorname{argmax}_{X \in C} P(X \mid Y)
$$


From Theorem 3, we know that the MAP estimate is optimal. In other words, MAP estimate gives an upper bound on what adversaries can achieve. Therefore, MAP estimate can be used to quantify the privacy.

In Equation (3), the expected accuracy value is computed for a particular $Y$ over all $X \in C$. To consider all $Y \in C$ from the disguised data set, we compute another expected value, but this time, over $Y$. This is the expected accuracy value for the entire disguised data. Namely, the value $A$ defined in Equation (2) can be computed using the following formula, rather than using the sample means:

$$
A=E_{Y}\left\{E_{X}\left[G\left(\widehat{X_{Y}}, X\right) \mid Y\right]\right\}=\sum_{Y \in C} P\left(\widehat{X_{Y}} \mid Y\right) \cdot P(Y)=\sum_{Y \in C} P\left(Y \mid \widehat{X_{Y}}\right) \cdot P\left(X_{Y}\right),
$$

where $\widehat{X_{Y}}$ is the MAP estimate for an given $Y$ and the last equality is from Bayes rules.

The value of $A$ represents the estimation accuracy from adversary's perspective; the larger $A$ is, the worse for privacy. Therefore, we define the BE privacy as $1-A$, i.e.,

$$
\mathcal{P}_{B E}=1-\sum_{Y \in C} P\left(Y \mid \widehat{X_{Y}}\right) \cdot P\left(X_{Y}\right)
$$

\section{Optimization of RR for Binary Data}

With the R-U maps, we can evaluate different RR schemes. Starting from the simplest, we take into account them for binary data (only two value $c_{1}, c_{2}$ in data), and present an approach to find the optimal $R R$ matrices with highest accuracy given certain privacy level. We denote the RR matrix $M$ to be

$$
M=\left(\begin{array}{ll}
\theta_{1,1} & \theta_{1,2} \\
\theta_{2,1} & \theta_{2,2}
\end{array}\right) .
$$

Because the sum of each column is 1 , a combination of the two elements $\theta_{1,1}$ and $\theta_{2,2}$ will determine the $M$.

We analyze distribution of the BE privacy along $\theta_{1,1}$ and $\theta_{2,2}$, which can be obtained from the following:

Theorem 4. When the BE privacy for binary data is less than $\min \{P(X=$ $\left.\left.c_{1}\right), P\left(X=c_{2}\right)\right\}$, it is $\mathcal{P}_{B E}=\left(1-\theta_{2,2}\right) P\left(X=c_{2}\right)+\left(1-\theta_{1,1}\right) P\left(X=c_{1}\right) \quad$ or $\mathcal{P}_{B E}=\theta_{1,1} P\left(X=c_{1}\right)+\theta_{2,2} P\left(X=c_{2}\right)$

If there is a coordinate system composed of variables $\theta_{1,1}$ and $\theta_{2,2}$, a BE privacy value $\left(<\min \left\{P\left(X=c_{1}\right), P\left(X=c_{2}\right)\right\}\right)$ is represented by a straight line in the coordinate system. Actually, 2 corresponding straight lines correspond to a privacy value and they are centrosymmetric with respect to the center point $(0.5,0.5)$.

After conducting many experiments, we find, in the optimal matrix with the highest BE privacy $\mathcal{P}=P\left(c_{1}\right)\left(P\left(c_{1}\right)<P\left(c_{2}\right)\right)$, some interesting rules, or equations or inequality between different elements: 
- $\theta_{1,1} * P\left(c_{1}\right)=\theta_{1,2} * P\left(c_{2}\right)$. This means that the proportion of $c_{1}$ randomized from original $c_{1}$ is the same as that from original $c_{2}$.

- $\theta_{1,1}>\theta_{2,2}$ and $\theta_{1,1}$ is as large as possible (here is 1 ). This results in the proportion of $c_{1}$ and that of $c_{2}$ in the randomized data being closer to each other.

The above two rules exist in the optimal $R R$ matrices for binary data and probably also exist in those for categorical data.

\section{Improved RR Scheme for Categorical Data}

After finding the optimal matrices for binary data which can achieve much better R-U map than Warner's scheme and observing the rules in an optimal matrix, we generalize the rules for categorical data. Based on the generalized rules together with another one obtained from other work, and under some constraint probably imposed by the data owners, we propose a heuristic approach which can find a RR matrix for categorical data achieving much better performance than the existing approaches. We introduce the rules and the constraint below.

\subsection{Rules and Constraint}

Rule 1. The first rule is from that used for binary data in the previous section. Suppose that the probabilities are sorted in an increasing order, i.e. $P(X=$ $\left.c_{1}\right) \leq \ldots \leq P\left(X=c_{n}\right)$. We have

$$
\theta_{r, r} * P\left(X=c_{r}\right)=\theta_{r, r+1} * P\left(X=c_{r+1}\right)=\ldots=\theta_{r, n} * P\left(X=c_{n}\right) .
$$

Rule 2. The second rule is also from that used for binary data. If there are $n$ different values $c_{1}, \ldots, c_{n}$ in a data set and their probabilities are in an incremental order, we have $\theta_{1,1} \geq \theta_{2,2} \geq \ldots \geq \theta_{n, n}$ in $M$.

However, when there are more than two categories in the data, the two rules can not be satisfied simultaneously. To control the tradeoff between the two rules, we introduce a variable, the upper bound of the difference between two neighbouring diagonal elements,

$$
E_{\text {dif }}, \quad \text { s.t. } \quad \theta_{i, i} \leq \theta_{i+1, i+1}+E_{\text {dif }},
$$

where $i=1, \ldots, n-1$. The variable will be used in our algorithm to find the optimal $M$.

Rule 3. From [5], the condition number is an important parameter to decide the estimation errors of the original probability distribution; the smaller the number is, the higher the estimation accuracy level. We wish the condition number of the optimal $M$ has an upper bound. Since the eigenvalues of an upper triangular matrix are the diagonal elements of the matrix, the lower bound of the condition number of a optimal $M$ is $\frac{\max _{i}\left(\theta_{i, i}\right)}{\min _{j}\left(\theta_{j, j}\right)}<\delta$, where $i, j=1, \ldots, n, \delta$ is a variable $(>1)$. 
Because the 1st diagonal element is usually 1, we simplify the above inequality as

$$
\theta_{j, j}>E_{\text {min }}, \text { for } j=1, \ldots, n,
$$

where $E_{\min }$ is a positive variable to control the condition number.

Constraint From Data Owners. Besides the rules for the optimal matrix, there could be a constraint imposed by the data owners. If any element in $M$ is 0 , e.g. $\theta_{1,3}=0$, the adversaries can immediately know that $c_{1}$ in randomized data must not come from $c_{3}$. This situation should be prevented. So the data owners might want to add a constraint which is $P\left(Y=c_{j} \mid X=c_{i}\right)>\sigma$, where $i, j=1, \ldots, n$ and $\sigma$ is a small number decided by the users.

Based on the rules and constraint, we propose an algorithm to find improved $\mathrm{RR}$ matrices for categorical data of a given probability distribution. We will show that the RR matrices achieve much better performance than Warner's scheme and FRAPP scheme [5] with experiments.

\subsection{Algorithm}

Suppose that the data set has $n$ categories and the probability distribution $\boldsymbol{P}$. For 2 specified variables $E_{\min }$ and $E_{\text {dif }}$, both of which can change from 0 to 1 , our algorithm is designed to find better RR matrices for the categorical data. The algorithm uses a brute force method, discretizing $E_{\min }$ and $E_{\text {dif }}$ due to certain step length and traversing all the combination of both variables to find the matrices. The brute force method has only 2 variables of the ranges from 0 to 1 so that it has much smaller search space than the brute force method used on all elements of an RR matrix $M$ which has $n^{2}$ variables of range from 0 to 1 . The algorithm is shown below.

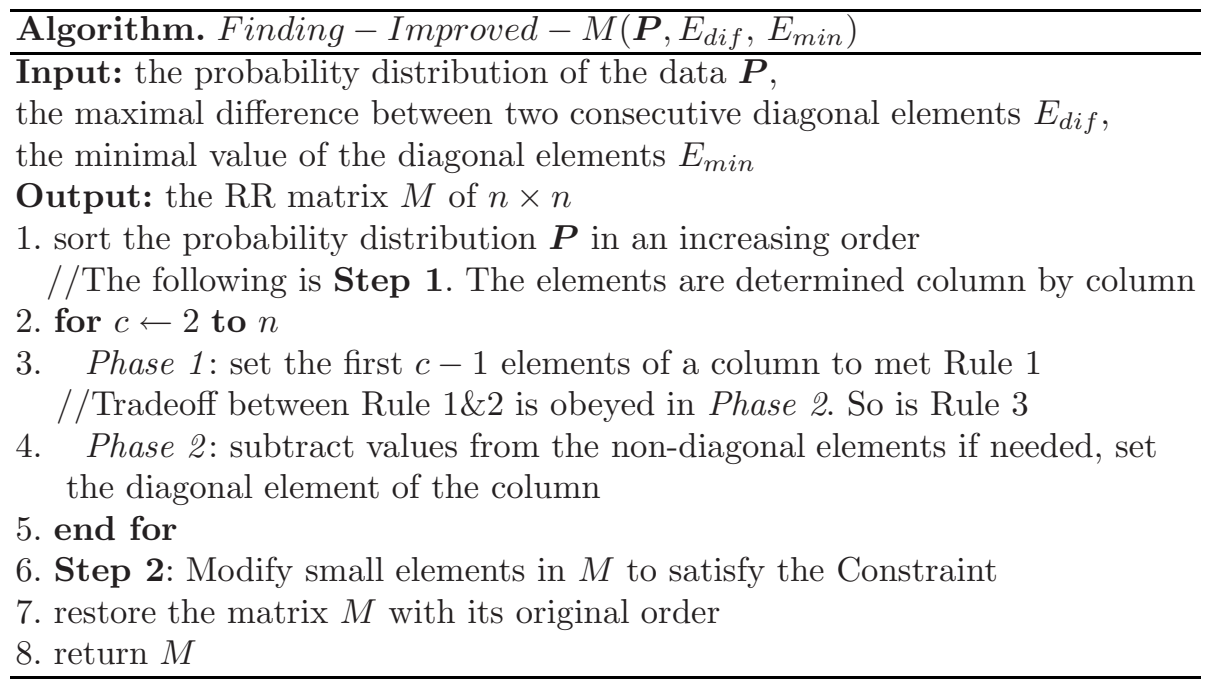


Complexity Analysis. Since in the main loop of the algorithm Phase 1 and Phase 2 all have computational overhead of $O(n)$, the loop has computational overhead of $O\left(n^{2}\right)$. The process of modifying the small elements has computational overhead of $O\left(n^{2}\right)$. So the total computational overhead is still $O\left(n^{2}\right)$. The space complexity is also $O\left(n^{2}\right)$ because of the used space for storing the matrix $M$. Suppose that $E_{\text {dif }}$ and $E_{\text {min }}$ are all discretized into $s$ different intervals, the whole algorithm has the computational overhead of $O\left(s^{2} \cdot n^{2}\right)$. But for the space overhead, if we want to store all the optimal matrix $M$, the total space overhead is $O\left(s^{2} \cdot n^{2}\right)$. If $s$ is not too large and data sets do not have too many categories ( $n$ is not very large), the algorithm is fast and does not consume much space.

\section{Experiments}

We design a series of experiments to compare our RR scheme and other RR schemes, i.e. the Warner's scheme and FRAPP [5]. Our experiments use real data, Adult data set in the UCI Machine Learning Repository [1] which has 14 attributes, including some continuous attributes. We discretize those continuous attributes in order to utilize any RR scheme. Because of the space limitation, we only show the results for the 1st attribute, but the results for other attributes have shown the same trends as the attribute.

The results in Figure 1 all show that our scheme has almost the same utility as Warner's scheme and the FRAPP scheme when BE privacy level is low while ours has much better utility (lower AMSE) than them when the BE privacy level becomes higher. This means that our scheme has much better performance than them especially when the BE privacy level is high.

The reason why our scheme achieves better performance than them is the following. An optimal class of RR matrices possess many rules, which are any equations or inequalities between different elements. The more rules the RR matrices generated with a scheme possess, the more similar to the optimal the generated matrices are. The more similar the RR matrices generated by a scheme is to the optimal, the better performance the scheme has. In our scheme, by following the rules and constraint, our generated RR matrices possess more rules than the matrices generated by other schemes, e.g. Warner's and FRAPP. So our scheme achieves better performance than them. 


\section{Conclusion}

In this paper, using R-U maps as a metric to systematically compare different $\mathrm{RR}$ schemes we present an approach to find an optimal class of RR matrices for binary data. In the optimal class, we discover some heuristic rules. By using the rules and a constraint probably imposed by the data owners, we propose a deterministic approach to finding a better class of RR matrices for categorical data. The experiments show that our scheme achieves much better performance than the existing RR schemes according to their R-U maps. Our future work is to continue searching for the optimal RR schemes for categorical data. We will also apply our scheme to some data mining computations to examine how utility is affected.

\section{References}

1. http://www.ics.uci.edu/ mlearn/MLRepository.html

2. Agrawal, D., Aggarwal, C.: On the design and quantification of privacy preserving data mining algorithms. In: Proccedings of the 20th ACM SIGACT-SIGMODSIGART Symposium on Principles of Database Systems, Santa Barbara, California, USA, ACM Press, New York (2001)

3. Agrawal, R., Srikant, R.: Privacy-preserving data mining. In: Proceedings of the 2000 ACM SIGMOD on Management of Data, pp. 439-450, Dallas, TX USA, May $15-8(2000)$

4. Agrawal, R., Srikant, R., Thomas, D.: Privacy preserving olap. In: Proceedings of the ACM SIGMOD Conference (SIGMOD 2005), Baltimore, Maryland, USA, June 14-16, 2005., ACM Press, New York (2005)

5. Agrawal, S., Haritsa, J.: A framework for high-accuracy privacy-preserving mining. In: Proceedings of 21st IEEE Intl. Conf. on Data Engineering (ICDE), Tokyo, Japan (2005)

6. Du, W., Zhan, Z.: Using randomized response techniques for privacy-preserving data mining. In: Proceedings of The 9th ACM SIGKDD International Conference on Knowledge Discovery and Data Mining, Washington, DC, USA, August 24-27, 2003 (2003)

7. Duncan, G.T., Keller-McNulty, S.A., Stokes, S.L.: Disclosure risk vs. data utility: The r-u confidentiality map. In: Technical Report Number 121, National Institute of Statistical Sciences (December 2001)

8. Evfimievski, A., Srikant, R., Agrawal, R., Gehrke, J.: Privacy preserving mining of association rules. In: Proceedings of 8th ACM SIGKDD International Conference on Knowledge Discovery and Data Mining, Edmonton, Alberta, Canada (July 2002)

9. Huang, Z.: Deriving private information from randomized data. In: Proceedings of the ACM SIGMOD Conference (SIGMOD 2005), Baltimore, Maryland, USA, June 14-16, 2005 (2005)

10. Kargupta, H., Datta, S., Wang, Q., Sivakumar, K.: On the privacy preserving properties of random data perturbation techniques. In: the IEEE International Conference on Data Mining, Melbourne, Florida, November 19 - 22, 2003, IEEE Computer Society Press, Los Alamitos (2003) 
11. Rizvi, S., Haritsa, J.R.: Maintaining data privacy in association rule mining. In: Proceedings of the 28th VLDB Conference, Hong Kong, China (2002)

12. Krishnan, V., Agrawal, S., Haritsa, J.R.: On addressing efficiency concerns in privacy-preserving mining. In: Lee, Y., Li, J., Whang, K.-Y., Lee, D. (eds.) DASFAA 2004. LNCS, vol. 2973, Springer, Heidelberg (2004)

13. Warner, S.L.: Randomized response: A survey technique for eliminating evasive answer bias. The American Statistical Association 60(309), 63-69 (1965) 